\title{
ALCALOIDES EN LA FLORA DE ANDALUCIA. II
}

\author{
Rafael SUAU, Ana I. GARCIA, Rodrigo RICO, \\ Baltasar CABEZUDO, José M NIETO-CALDERA \& Enrique SALVO
}

RESUMEN: Se han estudiado 105 especies vegetales características de la flora de Andalucía con objeto de determinar la presencia cualitativa y cuantitativa de alcaloides. Se ha encontrado presencia de alcaloides en 31 especies, de las cuales 19 se describen por primera vez.

Palabras clave: Alcaloides, flora de Andalucía, España.

SUMMARY: One hundred and five different species from the Andalusian flora have been screened for the presence of alkaloids. Of these, 31 species were found to contain alkaloids and 19 of then are reported for the first time.

Key words: Alkaloids, Andalusian flora, Spain.

\section{INTRODUCCION}

Continuando nuestras investigaciones sobre la presencia de alcaloides en plantas superiores de la flora de Andalucia (1) se describe, en este trabajo, el resultado del estudio de 105 especies que representan 74 géneros pertenecientes a 27 familias.

Fundamentalmente, se han analizado aquellas especies de las que no existen datos sobre su contenido en alcaloides. También se incluyen algunas especies que han sido ampliamente estudiadas por su contenido en alcaloides, pero que, dada la variabilidad cualitativa y cuantitativa que muestran, aconsejan un mayor conocimiento de las poblaciones que se desarrollan en Andalucía.

Especial atención se ha prestado a aquellas especies que se caracterizan por aparecer como plantas con valor terapeútico según la medicina popular tradicional, especialmente la que se ha desarrollado en el sur de Península Ibérica (2).

\section{METODOLOGIA}

El tratamiento del material vegetal, el aislamiento de la fracción de alcaloides y su determinación cuantitativa se han llevado a cabo de acuerdo con los procedimientos descritos anteriormente (1). 
La evaluación cualitativa de las bases terciarias y cuaternarias por los reactivos precipitantes de alcaloides se ha extendido con el empleo del reactivo Dragendorf, que ha mostrado especial utilidad con determinados géneros que contienen alcaloides pirrolicidínicos.

Análogamente a las consideraciones establecidas en el primer trabajo de esta serie, la reacción de precipitación se considera $[+++]$ cuando al adicionar los reactivos precipitantes se forma un precipitado abundante con o sin floculación y se considera $[++]$ cuando dicha adición forma una turbidez claramente definida sin floculación. En ambos casos se obtiene una conclusión positiva (P) sobre el contenido en alcaloides de la planta y la valoración cuantitativa con ácido p-toluen-sulfonico arroja un resultado igual o superior a $0.4 \mathrm{mEq} / 100 \mathrm{~g}$ de planta seca. La reacción es [+] cuando se forma una ligera, pero persistente, opacidad al adicionar los correspondientes reactivos. En este caso, la valoración define una conclusión positiva si es superior a $0.2 \mathrm{mEq}$ y si es igual o inferior a este valor se considera conclusión dudosa (D). Una reacción de precipitación muy ligera $[(+)]$ o su ausencia definen una conclusión negativa $(\mathrm{N})$.

\section{MATERIAL ESTUDIADO}

En la tabla II se indican, por orden alfabético, todas las especies estudiadas donde se establece, para cada una de ellas, lugar y fecha de recolección y número de registro de entrada en el herbario del Dpto. de Biología Vegetal de la Universidad de Málaga (MGC), donde todo el material se encuentra depositado como testigo.

\section{RES ULTADOS}

Los resultados obtenidos de la valoración de las distintas especies se representan en la tabla I, expresándose en $\mathrm{mEq}$ de alcaloides por cada $100 \mathrm{~g}$ de planta seca.

\section{CONCLUSIONES}

Se han estudiado 105 especies diferentes de las cuales 31 han dado resultados positivos, 13 dudosos, y 61 negativos. No se han encontrado estudios previos para 19 de las especies estudiadas.

Las nuevas especies que contienen alcaloides pertenecen a las familias Amarillidaceae, Astaraceae, Berberidaceae, Brasicaceae, Fabaceae, Lamiaceae, Liliaceae, Papaveraceae, Ranunculaceae, Resedaceae, Solanaceae, Valerianaceae y Violaceae.

Las familias que mayor contenido en alcaloides presentan son: Amarillidaceae, Berberidaceae, Fabaceae, Papaveraceae y Solanaceae. Resultado esperado puesto que todas ellas poseen géneros de alto contenido en alcaloides. 


\begin{tabular}{|c|c|c|c|c|c|c|}
\hline \multirow[t]{3}{*}{ FAMILIA-ESPECIE } & \multirow{3}{*}{\begin{tabular}{|c|} 
PARTE \\
DE LA \\
PLANTA
\end{tabular}} & \multicolumn{3}{|c|}{ ALCALOIDES } & \multirow{3}{*}{ CONCLUSION } & \multirow{3}{*}{$\begin{array}{l}\text { ESTUDIOS } \\
\text { PREVIOS }\end{array}$} \\
\hline & & \multicolumn{2}{|c|}{ TERCIARIOS } & \multirow{2}{*}{\begin{tabular}{|c} 
CUATERNARIOS \\
ENSAYOS \\
CUALITATIVOS
\end{tabular}} & & \\
\hline & & \begin{tabular}{|c|} 
ENSAYOS \\
CUALITATIVOS
\end{tabular} & \begin{tabular}{|c|} 
VALORACION \\
CUANTITATIVA
\end{tabular} & & & \\
\hline AMARYLLIDACEAE & & & & & & \\
\hline Leucojum autumnale (a) & PT & ++ & 1,5 & - & $\mathbf{P}$ & \\
\hline Leucojum autumnale (b) & PT & + & 1,2 & - & $\mathbf{P}$ & \\
\hline Narcissus cantabricus (a) & PT & + & 0,3 & - & $\mathbf{P}$ & \\
\hline $\begin{array}{c}\text { Narcissus cantabricus (b) } \\
\text { APIACEAE }\end{array}$ & PT & + & 0,7 & - & $\mathbf{P}$ & \\
\hline Bupleurum fruticosum & PA & $(+)$ & & - & $\mathbf{N}$ & 3 \\
\hline $\begin{array}{l}\text { Bupleurum gibraltaricum } \\
\text { ARISTOLOCHIACEAE }\end{array}$ & PA & $(+)$ & & - & N. & \\
\hline Aristolochia baetica & $\begin{array}{c}\mathrm{PA} \\
\mathrm{S}\end{array}$ & $\begin{array}{l}(+) \\
+\end{array}$ & 0,1 & - & $\mathrm{N}$ & 4 \\
\hline $\begin{array}{r}\text { Aristolochia paucinervis } \\
\text { ASTERACEAE }\end{array}$ & PA & $(+)$. & 0,1 & $(+)$ & $\mathrm{N}$ & 5 \\
\hline Artemisia herba-alba & PT & $(+)$ & & - & $\mathrm{N}$ & 6 \\
\hline Cladanthus arabicus (a) & PT & $(+)$ & & - & $\mathrm{N}$ & \\
\hline Cladanthus arabicus (b) & PT & t+ & 0,2 & - & D & \\
\hline $\begin{array}{l}\text { Helichrysum italicum } \\
\text { subsp. serotinum }\end{array}$ & PA & + & 0,2 & - & D & \\
\hline $\begin{array}{l}\text { Jurinea pinnata } \\
\text { BERBERIDACEAE }\end{array}$ & PA & ++ & 0,7 & $(+)$ & $\mathbf{P}$ & \\
\hline $\begin{array}{l}\text { Berberis hispanica } \\
\text { BORAGINACEAE }\end{array}$ & $\mathbf{R}$ & +++ & 8,0 & +++ & $\mathbf{P}$ & $7-8$ \\
\hline Buglossoides arvensis & PT & - & & - & $\mathbf{N}$ & \\
\hline Echium boissieri & PA & - & & - & $\mathbf{N}$ & \\
\hline Echium creticum & & & & & & \\
\hline subsp. coincyanum (a) & PA & $(+)$ & & - & $\mathbf{N}$ & \\
\hline Echium creticum & & & & & & \\
\hline subsp. coincyanum (b) & PA & - & & - & $\mathrm{N}$ & \\
\hline Echium plantagineum (a) & PA & $(+)$ & & - & $\mathbf{N}$ & $7-9-10-11$ \\
\hline Echium plantagineum (b) & PA & - & & - & $\mathrm{N}$ & $"$ \\
\hline Echium plantagineum (c) & PA & - & & - & $\mathrm{N}$ & $"$ \\
\hline Echium plantagineum (d) & PA & - & & - & $\mathrm{N}$ & $"$ \\
\hline Echium plantagineum (e) & PA & $(+)$ & & - & $\mathrm{N}$ & $"$ \\
\hline Echium plantagineum ( $f$ ) & PA & $(+)$ & & - & $\mathbf{N}$ & $"$ \\
\hline & $\mathbf{R}$ & - & & - & $\mathbf{N}$ & $"$ \\
\hline Lithodora fruticosa (a) & PA & - & & - & $\mathrm{N}$ & \\
\hline Lithodora fruticosa (b) & PA & - & & - & $\mathrm{N}$ & \\
\hline Lithodora prostrata & & & & & & \\
\hline subsp. lusitanica (a) & PA & - & & - & $\mathbf{N}$ & \\
\hline Lithodora prostrata & & & & & & \\
\hline $\begin{array}{l}\text { subsp. lusitanica (b) } \\
\text { BRASSICACEAE }\end{array}$ & PA & - & & - & $\mathrm{N}$ & \\
\hline Biscutella frutescens & PA & + & 0,3 & - & $\mathbf{P}$ & \\
\hline Brassica barrelieri & PT & - & & - & $\mathrm{N}$ & \\
\hline Brassica repanda & & & & & & \\
\hline subsp.latisiliqua & PA & + & 0,2 & + & D & \\
\hline Descurainia sophia & PT & +t & 0,6 & +t & $\mathrm{P}$ & $9-15$ \\
\hline Diplotaxis crassifolia & PA & - & & - & $\mathrm{N}$ & \\
\hline Hesperis laciniata & PA & + & 0,6 & $(+)$ & $\mathrm{P}$ & \\
\hline Iberis nazarita & PA & + & 0,2 & $(+)$ & D & 1 \\
\hline $\begin{array}{l}\text { Lobularia maritima } \\
\text { CAMPANULACEAE }\end{array}$ & PT & $(+)$ & & - & $\mathbf{N}$ & 6 \\
\hline $\begin{array}{l}\text { Campanula velutina } \\
\text { CIST ACEAE }\end{array}$ & PA & - & & - & $\mathrm{N}$ & \\
\hline Halimium halimifolium & PA & - & & - & $\mathrm{N}$ & \\
\hline Halimium lasianthum & PA & $(+)$ & & - & $\mathrm{N}$ & \\
\hline $\begin{array}{l}\text { Helianthemum lavandulifolium } \\
\text { CLUSI ACEAE }\end{array}$ & PA & - & & - & $\mathrm{N}$ & \\
\hline Hypericum perforatum & PA & - & & - & $\mathrm{N}$ & \\
\hline
\end{tabular}




\begin{tabular}{|c|c|c|c|c|c|c|}
\hline \multirow[t]{3}{*}{ FAMILIA-ESPECIE } & \multirow{3}{*}{\begin{tabular}{|c|} 
PARTE \\
DE LA \\
PLANTA
\end{tabular}} & \multicolumn{3}{|c|}{ ALCALOIDES } & \multirow{3}{*}{ CONCLUSION } & \multirow{3}{*}{\begin{tabular}{|l|} 
ESTUDIOS \\
PREVIOS
\end{tabular}} \\
\hline & & \multicolumn{2}{|c|}{ TERCIARIOS } & CUATERNARIOS & & \\
\hline & & $\begin{array}{c}\text { ENSAYOS } \\
\text { CUALITATIVOS }\end{array}$ & $\begin{array}{c}\text { VALORACION } \\
\text { CUANTITATIVA }\end{array}$ & \begin{tabular}{|c|} 
ENSAYOS \\
CUALITATIVOS
\end{tabular} & & \\
\hline CORIARIACEAE & & & & & & \\
\hline Coriaria myrtifolia (a) & PA & $(+)$ & & - & $\mathbf{N}$ & 12 \\
\hline Coriaria myntifolia (b) & PA & - & & - & $\mathrm{N}$ & 12 \\
\hline Coriaria myrtifolia (c) & PA & - & & - & $\mathrm{N}$ & 12 \\
\hline $\begin{array}{l}\text { ERICACEAE } \\
\text { Calluna vulgaris }\end{array}$ & PA & - & & 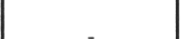 & $\mathbf{N}$ & \\
\hline Rhododendron ponticum & & - & & 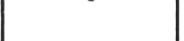 & IN & \\
\hline $\begin{array}{l}\text { subsp. baeticum } \\
\text { EUPHORIACE }\end{array}$ & PA & - & & - & $\mathrm{N}$ & \\
\hline Euphorbia betica & PA & - & & - & $\mathrm{N}$ & \\
\hline $\begin{array}{r}\text { Mercurialis tomentosa } \\
\text { FABACEAE }\end{array}$ & PA & - & & - & $\mathrm{N}$ & \\
\hline Cytisus baeticus & PA & +++ & 3,3 & $(+)$ & $\mathbf{P}$ & \\
\hline Cytisus grandiflorus (a) & PA & t++ & 3,8 & ++ & $\mathbf{P}$ & 13 \\
\hline Cytisus grandiflorus (b) & PA & t++ & 5,0 & + & $\mathbf{P}$ & 13 \\
\hline Cytisus grandiflorus (c) & PA,F & t++ & 2,5 & + & $\mathbf{P}$ & 13 \\
\hline Cytisus villosus (a) & PA & t++ & 1,8 & + & $\mathbf{P}$ & \\
\hline Cytisus villosus (b) & PA & +++ & 2,0 & + & $\mathbf{P}$ & \\
\hline Dorycnium pentaphyllum & PA & - & & - & $\mathrm{N}$ & 12 \\
\hline Dorycnium rectum & PA & $(+)$ & & - & $\mathrm{N}$ & $12-14$ \\
\hline Echinospartum boissieri & PA & - & & - & $\mathrm{N}$ & \\
\hline Genista monspessulana & PA,F & +++ & 3,4 & + & $\mathbf{P}$ & \\
\hline Lotus boissieri & PA & + & 0,3 & - & $\mathbf{P}$ & \\
\hline Ononis speciosa & PA & + & 0,2 & - & D & \\
\hline FRANKENIACEAE & & & & & & \\
\hline $\begin{array}{l}\text { Frankenia thymifolia } \\
\text { IRIDACEAE }\end{array}$ & PA & - & & - & $\mathbf{N}$ & \\
\hline Crocus serotinus & & & & & & \\
\hline subsp. salzmannii & PT & - & & - & $\mathrm{N}$ & \\
\hline Iris planifolia (a) & PT & - & & - & $\mathrm{N}$ & \\
\hline Iris planifolia (b) & PT & - & & - & $\mathrm{N}$ & \\
\hline Iris pseudacorus & PT & - & & - & $\mathrm{N}$ & $14-15-16$ \\
\hline $\begin{array}{l}\text { Romulea bulbocodium } \\
\text { LAMIACEAE }\end{array}$ & PT & - & & - & $\mathbf{N}$ & \\
\hline Lycopus europacus & PA & - & & - & $\mathrm{N}$ & 7 \\
\hline Marrubium supinum & PA & + & 0,5 & - & $\mathbf{P}$ & \\
\hline Mentha aquatica & PT & - & & - & $\mathrm{N}$ & \\
\hline Mentha suaveolens & PT & - & & - & $\mathrm{N}$ & \\
\hline Nepeta amethystina & & & & & & \\
\hline subsp. laciniata & PA & + & 0,3 & - & $\mathbf{P}$ & \\
\hline Nepeta tuberosa & & $t$ & & - & & \\
\hline subsp. tuberosa & PA & + & 0,1 & - & D & \\
\hline Phlomis crinita & $\begin{array}{l}\text { PA } \\
\text { PA }\end{array}$ & $\begin{array}{l}- \\
-\end{array}$ & & $\begin{array}{l}- \\
-\end{array}$ & $\begin{array}{l}\mathrm{N} \\
\mathrm{N}\end{array}$ & \\
\hline Phlomis herba-venti & $\begin{array}{l}\text { PA } \\
\text { PA }\end{array}$ & - & & - & $\mathrm{N}$ & 3 \\
\hline Phlomis lychnitis & $\begin{array}{l}\text { PA } \\
\text { PA }\end{array}$ & - & & - & $\mathrm{N}$ & \\
\hline Phlomis purpurea & $\begin{array}{l}\text { PA } \\
\text { PA }\end{array}$ & - & & - & $\mathbf{N}$ & \\
\hline $\begin{array}{l}\text { Stachys circinata } \\
\text { Teucrium lusitanicum }\end{array}$ & $\begin{array}{l}\text { PA } \\
\text { PT }\end{array}$ & - & & - & $\mathrm{N}$ & \\
\hline $\begin{array}{l}\text { Teucrium lusitanicum } \\
\text { Teucrium luteum }\end{array}$ & PA & + & 0,1 & (t) & D & \\
\hline Teucrium scordium & & & & & & \\
\hline subsp. scordioides & PA & - & & - & $\mathrm{N}$ & \\
\hline LILIACEAE & PT & + & 0.5 & - & & \\
\hline $\begin{array}{l}\text { Allium chamaemoly } \\
\text { Allium subvillosum }\end{array}$ & PT & + & 0,4 & - & $\begin{array}{l}\mathrm{P} \\
\mathrm{P}\end{array}$ & \\
\hline $\begin{array}{l}\text { Allium subvillosum } \\
\text { Asparagus acutifolius }\end{array}$ & $\mathrm{PA}$ & $(+)$ & & - & $\begin{array}{l}\mathrm{P} \\
\mathrm{N}\end{array}$ & 3 \\
\hline $\begin{array}{l}\text { Asparagus acutifolius } \\
\text { Colchicum lusitanum }\end{array}$ & PT & ++ & 0,3 & - & $\begin{array}{l}\text { P } \\
\text { P }\end{array}$ & $\begin{array}{l}3 \\
7\end{array}$ \\
\hline $\begin{array}{l}\text { Colchicum lusitanum } \\
\text { Dipcadi serotinum }\end{array}$ & PT & - & & - & $\mathrm{N}$ & 3 \\
\hline $\begin{array}{l}\text { Dipcadi serotinum } \\
\text { Fritillaria lusitanica }\end{array}$ & PT & - & & - & $\mathrm{N}$ & \\
\hline $\begin{array}{l}\text { Fritillaria lusitanica } \\
\text { Gagea nevadensis }\end{array}$ & PT & - & & - & $\mathrm{N}$ & \\
\hline $\begin{array}{l}\text { Gagea nevadensis } \\
\text { Hyacinthoides hispanica }\end{array}$ & PT & - & & - & $\mathrm{N}$ & \\
\hline $\begin{array}{l}\text { Hyacinthoides hispanica } \\
\text { Muscari comosum }\end{array}$ & PT & - & & - & $\mathrm{N}$ & 17 \\
\hline
\end{tabular}

Tabla I (Continuación) 


\begin{tabular}{|c|c|c|c|c|c|c|}
\hline \multirow[t]{3}{*}{ FAMLIA-ESPECIE } & \multirow{3}{*}{\begin{tabular}{|c|} 
PARTE \\
DE LA \\
PLANTA
\end{tabular}} & \multicolumn{3}{|c|}{ ALCALOIDES } & \multirow[t]{3}{*}{ CONCLUSION } & \multirow{3}{*}{$\begin{array}{l}\text { ESTUDIOS } \\
\text { PREVIOS }\end{array}$} \\
\hline & & \multicolumn{2}{|c|}{ TERCIARIOS } & $\begin{array}{l}\text { CUATERNARIOS } \\
\end{array}$ & & \\
\hline & & $\begin{array}{c}\text { ENSAYOS } \\
\text { CUALITATIVOS }\end{array}$ & \begin{tabular}{|c|} 
VALORACION \\
CUANTITATIVA \\
\end{tabular} & \begin{tabular}{|c|} 
ENSAYOS \\
CUALITATIVOS \\
\end{tabular} & & \\
\hline LILIACEAE (cont.) & & & & & & \\
\hline Ornitogalum narbonense & PA & + & 0,1 & + & D & \\
\hline Scilla autumnalis (a) & PT & - & & - & $\mathrm{N}$ & 9 \\
\hline Scilla autumnalis (b) & PT. & - & & - & $\mathrm{N}$ & 9 \\
\hline Tulipa silvestris & & & & & & \\
\hline subsp. australis & PT & - & & - & $\mathrm{N}$ & \\
\hline $\begin{array}{l}\text { Urginea maritima } \\
\text { PAEONIACEAE }\end{array}$ & PT & + & 0,2 & + & D & \\
\hline $\begin{array}{l}\text { Paconia broteroi } \\
\text { PAPAVERACEAE }\end{array}$ & PA & - & & - & $\mathrm{N}$ & \\
\hline Platycapnos saxicola & $\mathrm{PA}$ & t+t & & + & $\mathrm{P}$ & \\
\hline Platycapnos spicata & PT & t+t & 4,3 & ++ & $\mathrm{P}$ & 18 \\
\hline Platycapnos tenuiloba & & & & & & \\
\hline - subsp.parallela & PT & +++ & 4,0 & - & $\mathrm{P}$ & \\
\hline Platycapnos tenuiloba & & & & & & \\
\hline subsp. tenuiloba & PT & +++ & 4,0 & + & $\mathrm{P}$ & \\
\hline $\begin{array}{l}\text { RANUNCULACEAE } \\
\text { Anemone palmata }\end{array}$ & PT & - & & - & $\mathrm{N}$ & \\
\hline Clematis flammula & PA & - & & - & $\mathrm{N}$ & 3 \\
\hline Delphinium gracile & PT & t+ & 1,0 & + & $\mathrm{P}$ & \\
\hline Helleborus foetidus & PT & - & & - & $\mathrm{N}$ & 12 \\
\hline Ranunculus bullatus & PT & $(+)$ & & - & $\mathrm{N}$ & \\
\hline $\begin{array}{l}\text { Ranunculus paludosus } \\
\text { RESEDACEAE }\end{array}$ & PT & $(+)$ & & - & $\mathrm{N}$ & \\
\hline Reseda complicata & PA & - & & - & $\mathrm{N}$ & \\
\hline Reseda phyteuma & PA & + & 0,4 & - & $P$ & \\
\hline Osyris alba & PT & + & 0,2 & - & D & \\
\hline Viscum cruciatum (a) & $\mathrm{PA}$ & - & & - & $\mathrm{N}$ & 9 \\
\hline Viscum cruciatum (b) & $\mathrm{PA}$ & - & & - & $\mathrm{N}$ & 9 \\
\hline SOLANACEAE & & & & & & \\
\hline Lycium intrincatum & $\mathrm{PA}$ & + & 0,2 & ++ & D & \\
\hline Mandragora autumnalis & PT & +t & 0,3 & ++ & $\mathrm{P}$ & \\
\hline $\begin{array}{l}\text { Withania frutescens } \\
\text { THELGONACEAE }\end{array}$ & $\therefore \mathrm{PA}$ & + & 0,3 & + & $\mathrm{P}$ & 9 \\
\hline $\begin{array}{l}\text { THELIGONACEAE } \\
\text { Theligonum cynocrambe }\end{array}$ & $\mathrm{PA}$ & + & 0,1 & - & D & \\
\hline VALERIANACEAE & & & & - & & \\
\hline Centranthus macrosiphon & PT & + & 0,4 & $\therefore$ & $\mathrm{P}$ & \\
\hline Valeriana tuberosa & PT & $(+)$ & & $(+)$ & $\mathrm{N}$ & \\
\hline $\begin{array}{l}\text { VIOLACEAE } \\
\text { Viola crassiuscula }\end{array}$ & PT & + & 0,3 & & $\mathbf{P}$ & \\
\hline
\end{tabular}

Tabla I (Continuación)

Aunque Lamiaceae no se caracterizan por sus alcaloides, se ha confirmado la presencia de los mismos en el género Nepeta y la valoración positiva en el género Marrubium.

El estudio de distintas especies de Echium y Lithodora ha dado en todos los casos resultados negativos, a pesar de que la familia Boraginaceae suelen presentar alcaloides pirrolizidínicos, aunque en bajas concentraciones (10). Así mismo se han obtenido resultados negativos en todas las especies estudiadas hasta ahora de familias como Coriaceae, Ericaceae, Euphorbiaceae e Iridaceae.

Por otra parte, se ha confirmado la ausencia de alcaloides en algunos géneros que inicialmente dieron resultados dudosos. Así, por ejemplo, del género Bupleurum, perteneciente a la familia Apiaceae, se han estudiado 3 especies todas ellas con resultados negativos, sugiriendo que el resultado obtenido anteriormente para la especie $B$. spinosum (1), se trate de un falso dudoso. Otro ejemplo es el género Teucrium (Lamiaceae) donde, de las 6 especies estudiadas hasta el momento, solamente 2 han registrado resultados dudosos con cantidades inferiores a 0.2 $\mathrm{mEq} / 100 \mathrm{~g}$ de planta seca lo que indica un contenido en alcaloides negativo en este género. 
El estudio de las raíces de Berberis hispanica (Berberidaceae) muestra una concentración en alcaloides 20 veces superior a la de las partes aereas (1). Esto era de esperar, pues este género es conocido por su alto contenido en alcaloides.

El género Aristolochia se caracteriza por la presencia de ácidos aristolóquicos y aristolactamas, compuesto de reconocida actividad anticancerígena. Sin embargo, es de destacar la ausencia de alcaloides aporfínicos que son los precursores biosintéticos de dichos ácidos. Incluso en el estudio de las semillas de la $A$. baetica, lugar de la planta donde suelen concentrarse estos alcaloides, los resultados no han sido positivos.

Sin embargo, los alcaloides aporfínicos son los principales constityentes del género Platycapnos (Papaveraceae), del que se han estudiado 4 especies diferentes de las cuales la primera de ellas, $P$. saxicola, no se ha podido cuantificar por disponer solamente de $2 \mathrm{~g}$ de planta seca, cuyo extracto alcaloidico, sin embargo, reaccionó fuertemente frente a los reactivos precipitantes de alcaloides. Todas las especies estudiadas de este género poseen un contenido en alcaloides entre 4 y $5 \mathrm{mEq}$.

\section{AGR ADECIMIENTOS}

Este trabajo ha sido financiado por la Consejería de Educación y Ciencia de la Junta de Andalucía.

\section{BIBLIOGR AFIA}

1.- SUAU, R., GARCIA, A.I., RICO, R., CABEZUDO, B., NIETO, J.M. \& SALVO, E. -1988- Alcaloides en la Flora de Andalucía. I. Acta Botánica Malacitana 13:189.

2.- LAZA, M. -1940- Florula Malacitana. Anales Real Acad. de Farmacia. 165.

3.- SERRANO, M., CODINA, C., VILADOMAT, F., BASTIDA, J. \& LLABRES, J.M. 1985- Alkaloid Screening of Catalonia (Spain) plants. II. Int. J. Crude Drug Res. 23:105-107.

4.- CARRERAS, L.M. -1973- Bases cuatemarias y nitrofenantrenos en Aristolochia baetica L. Anal. Inst. Bot. Cavanillas 30:253-265.

5.-PASCUAL, J., URONES, J. \& FERNANDEZ, A. -1983- An Aristolochic acid derivative from Aristolochia longa. Phytochemistry, 23:2745-49.

6.- SMOLENSKI, S.J., SILINIS, H. \& FARNSWORTS, N.R. -1974- Alkaloid Screening. V. Lloydia 37:506.

7.- WILLAMAN, J.J. \& LI, H.L. -1970- Alkaloid-bearing plants and their contained alkaloids. 1957-1968. Lloydia 33, suppl. 3A.

8.- SUAU, R. \& CUEVAS, A. Tesis doctoral (En preparación). Málaga.

9.- SMOLENSKI, S.J., SILINIS, H. \& FARNSWORTH, N.R. -1972- Alkaloid Screening. I. Lloydia 35:1.

10.- MATTOCKS, A.R. -1986- Chemistry and Toxicology of Pyrrolizidine Alkaloids. Ed. Academic Press. London.

11.- CULVENOR, C.C.J. -1956- The alkaloids of Echium plantagineum,Aust. J. Chem. 9: $512-514$.

12.- VILADOMAT, F., CODINA, C., BASTIDA, J., GALOBARDES, M. \& SERRANO, M. -1984- Alkaloid Screening of Catalonia (Spain) Plants. I. Lloydia 47:64-69.

13.- WHITE, E. -1943- Chemotaxonomy of the Leguminosae. N.Z. Jl. Sci. Technol. B 25: 93.

14.- SMOLENSKI, S.J., SILINIS, H. \& FARNSWORTH, N.R. -1975- Alkaloid Screening. IV. Lloydia 38:225. 
15.- SMOLENSKI, S.J., SILINIS, H. \& FARNSWOETH, N.R. -1973- Alkaloid Screening. III. Lloydia 36:359.

16.- FONG, H.H.S., TROJANKOVA, M., TROJANEK, J. \& FARNSWORTH, N.R. 1972- Alkaloid Screening. II. Lloydia 35: 117.

17.- VILADOMAT, F., CODINA, C., LLABRES, J.M. \& BASTIDA, J. -1986- Alkaloid Screening of Plants of Catalonia (Spain). III. Int. J. Crude Drug Res. 24:123-130.

18.- GARCIA MARIN, A.I. -1985- Aislamiento, Determinación Estructural y Síntesis de los alcaloides del Platycapnos spicata subsp. spicata. Tesis de Licenciatura. Universidad de Málaga.

\section{ADDENDA I}

\section{MATERIAL ESTUDIADO}

Allium chamaemoly L., Cádiz: Algeciras; 28.XI.86 (MGC 18204)

Allium subvillosum Salzm. ex Schultes \& Schultes, Granada: Motril; 18.IV.86 (MGC 21367)

Anemone palmata L., Málaga: Istán; 25.IV.86 (MGC 21529)

Aristolochia baetica L., Málaga: Málaga; 6.XII.87 (MGC 20728)

Aristolochia paucinervis Pomel., Sevilla: Valdeflores; 4.IV.86 (MGC 20727)

Artemisia herba-alba Asso., Granada: Cúllar-Baza; 27.IV.86 (MGC 21370)

Asparagus acutifolius L., Málaga: Alhaurín el Grande; 16.III.86 (MGC 20730)

Berberis hispanica Boiss. \& Reuter (b), Granada: Sierra Tejeda; 27.III.87 (MGC 21603)

Biscutella frutescens Cosson, Málaga: Sierra Tejeda; 27.III.87 (MGC 21599)

Brassica barrelieri (L.) Janka, Huelva: Mazagón; 6.IV.86 (MGC 21379)

Brassica repanda (Willd.) DC. subsp. latisiliqua (Boiss. \& Reuter) Heywood, Málaga: Cómpeta; 26.VI.86 (MGC 23002)

Buglossoides arvensis (L.) I.M. Johnston, Granada: Guadix; 27.IV.86 (MGC 21339)

Bupleurum fruticosum L., Huelva: Linares de la Sierra; 4.IV.86 (MGC 20731)

Bupleurum gibraltaricum Lam., Málaga: Alpandeire; 27.III.88 (MGC 21590)

Calluna vulgaris (L.) Hull., Cádiz: Algeciras; 28.XI.86 (MGC 18201)

Campanula velutina Desf., Málaga: Alhaurín el Grande; 16.II.86 (MGC 20707)

Centranthus macrosiphon Boiss., Málaga: El Burgo; 27.III.88 (MGC 21558)

Cladanthus arabicus (L.) Cass. (a), Málaga: Sierra de Mijas; 16.III.86 (MGC 23004)

Cladanthus arabicus (L.) Cass. (b), Málaga: Campanillas; 1.III.88 (MGC 21563)

Clematis flammula L., Granada: Zafarraya; 18.IX.87 (MGC 20653)

Colchicum lusitanum Brot., Málaga: Alora; 11.X.86 (MGC 23013)

Coriaria myrtifolia L. (a), Málaga: Istán; 25.IV.86 (MGC 20720)

Coriaria myrtifolia L. (b), Málaga: Istán; 12.IV.88 (MGC 23026)

Coriaria myrtifolia L. (c), Málaga: Tolox; 2.VI.87 (MGC 20700)

Crocus serotinus Salisb. subsp. salzmannii (J. Gay) Mathew., Málaga: Almogía; 24.X.86 (MGC 22994).

Cytisus baeticus (Webb) Steudel, Málaga: Cortes de la Frontera; 28.III.88 (MGC 21594)

Cytisus grandiflorus DC. (a), Huelva: Almonte; 6.IV.86 (MGC 21376)

Cytisus grandiflorus DC. (b), Málaga: Benahavis; 20.II.88 (MGC 21598)

Cytisus grandiflorus DC. (c), Málaga: Istán; 2.VI.87 (MGC 21624)

Cytisus villosus Pourret (a), Málaga: Istán; 5.II.88 (MGC 21595)

Cytisus villosus Pourret (b), Málaga: Benahavis; $20 \Pi 188$ (MGC 21609)

Delphinium gracile DC., Huelva: Almonte; 8.IX.87 (MGC 18250)

Descurainia sophia (L.) Webb ex Pranttl., Granada: Guadix; 27.IV.86 (MGC 23003)

Dipcadi serotinum (L.) Medicus, Málaga: Alora; 22.III.87 (MGC 23017)

Diplotaxis crassifolia (Rafin) DC., Almería: entre Sorbas y Lubrín; 4.VI.86 (MGC 21533)

Dorycnium pentaphyllum Scop., Almería: Tabernas; 4.VI.86 (MGC 22997)

Dorycnium rectum (L.) Ser. in DC., Málaga: Tolox; 2.VI.87 (MGC 20599)

Echinospartum boissieri (Spach) Rothm., Málaga: Cómpeta; 26.VI.86 (MGC 21366) 
Echium boissieri Steudel, Málaga: Alhauurín de la Torre; 2.VI.87 (MGC 21575)

Echium creticum L. subsp. coincyanum (Lacaita) R. Fernández (a), Málaga: Istán; 2.VI.87 (MGC 21576)

Echium creticum L. subsp. coincyanum (Lacaita) R. Fernández (b), Málaga: Coín; 2.VI.87 (MGC 21573)

Echium plantagineum L. (a), Málaga: Alhaurín de la Torre; 2.VI.87 (MGC 21578)

Echium plantagineum L. (b), Málaga: Coín; 2.VI.87 (MGC 21572)

Echium plantagineum L.(c), Málaga; Torrox; 2.VI.87 (MGC 21574)

Echium plantagineum L. (d), Málaga: Monda; 2.VI.87 (MGC 21579)

Echium plantagineum L. (e), Málaga: Tolox; 2.VI.87 (MGC 21571)

Echium plantagineum L. (f), Málaga: Málaga; 6.XII.87 (MGC 21573)

Euphorbia baetica Boiss., Huelva: Almonte; 6.IV.86 (MGC 21377)

Frankenia thymifolia Desf., Almería: Huercal-Overa; 4.VI.86 (MGC 21627)

Fritillaria lusitanica Wikström, Málaga: Alora, 22.III.86 (MGC 23020)

Gagea nevadensis Boiss., Málaga: Alhaurín de la Torre; 23.II.86 (MGC 20662)

Genista monspessulana (L.) L. Johnson (b), Cádiz: Algeciras; 22.IV.88 (MGC 23024)

Halimium halimifolium (L.) Willk., Huelva: Almonte; 6.IV.86 (MGC 21361)

Halimium lasianthum (Lam.) Spach., Cádiz: Cortes de la Frontera; 8.XI.86 (MGC 18208)

Helianthemum lavandulifolium Miller, Granada: Albuñol; 11.VI.88 (MGC 22999)

Helichrysum italicum (Roth.) G. Don fil. subsp. serotinum (Boiss.) P. Fourn., Granada: Sierra Nevada; 22.VII.87 (MGC 18242)

Helleborus foetidus L. (a), Málaga: Tolox; 25.IV.86 (MGC 20722)

Hesperis laciniata All., Málaga: Sierra Tejeda; 27.III.87 (MGC 21591)

Hyacinthoides hispanica (Miller) Rothm., Huelva: Linares de la Sierra: 4.IV.86 (MGC 20732)

Hypericum perforatum L., Málaga: Cómpeta; 26.VI.87 (MGC 23029)

Iberis nazarita Moreno, Málaga: Coín; 2.VI.87 (MGC 23028)

Iris planifolia (Miller) Fiori \& Paol. (a), Málaga: El Burgo; 9.II.86 (MGC 20660)

Iris planifolia (Miller) Fiori \& Paol (b), Málaga: Alora; 17.I.87 (MGC 22993)

Iris pseudacorus L., Huelva: Almonte; 6.IV.86 (MGC 21362)

Jurinea pinnata (Lag.) DC., Málaga: Yunquera; 7.VI.86 (MGC 21359)

Leucojum autumnale L. (a), Málaga: entre Almogía y Antequera; 24.X.86 (MGC 18211)

Leucojum autumnale L. (b), Málaga: Coín; 8.X.87 (MGC 20652)

Lithodora fruticosa (L.) Griseb. (a), Granada: Sierra Tejeda; 27.III.87 (MGC 21601)

Lithodora fruticosa (L.) Griseb. (b), Málaga: El Burgo; 27.III.88 (MGC 21589)

Lithodora prostrata (Loisel.) Griseb. subsp. lusitanica (Samp.) Valdés (a), Málaga: Coín; 1.III.88 (MGC 21565)

Lithodora prostrata (Loisel.) Griseb. subsp. lusitanica (Samp.) Valdés (b), Málaga: Tolox; 5.II.88 (MGC 21547)

Lobularia maritima (L.) Desv., Málaga: Alhaurín de la Torre; 16.III.86 (MGC 20713)

Lotus boissieri Fernandes, Granada: Sierra Nevada; 23.VII.87 (MGC 20630)

Lycium intricatum Boiss., Málaga: Nerja; 28.II.86 (MGC 20706)

Lycopus europaeus L., Huelva: Almonte; 9.IX.87 (MGC 18240)

Mandragora autumnalis Bertol., Málaga: Villanueva de la Concepción; 24.X.86 (MGC 18209)

Marrubium supinum L., Granada: Sierra Nevada; 22.VII.87 (MGC 20642)

Mentha aquatica L., Huelva: Almonte; 9.IX.97 (MGC 18248)

Menta suaveolens Ehrh., Huelva: Almonte; 9.IX.87 (MGC 18239)

Mercurialis tomentosa L., Málaga: Yunquera; 7.VI.86 (MGC 21375)

Muscari comosum (L.) Miller, Grànada: Motril; 18.IV.86 (MGC 21538)

Narcissus cantabricus DC. (a), Málaga: Alora; 1.II.87 (MGC 22994)

Narcissus cantabricus DC. (b), Málaga: Istán; 20.II.88 (MGC 21592)

Nepeta amethystina Poiret subsp. laciniata (Willk.) Ubera \& Valdés, Granada: Sierra Nevada; 23.VII.87 (MGC 18264)

Nepeta tuberosa L. subsp. tuberosa, Málaga: Cómpeta; 26.VI.86 (MGC 20686)

Ononis speciosa Lag. (b), Málaga: Alpandeire; 27.III.88 (MGC 21555)

Ornithogalum narbonense L., Málaga: Alora; 4.V.87 (MGC 23019) 
Osyris alba L., Huelva: Valverde del Camino;4.IV.86 (MGC 20733)

Paeonia broteroi Boiss. \& Reuter, Granada: Sierra Tejeda; 27.III.87 (MGC 21602)

Phlomis crinita Cav., Málaga: Yunquera; 7.VI.86 (MGC 20682)

Phlomis herba-venti L., Málaga: Alora; 21.VI.86 (MGC 20688)

Phlomis lychnitis L., Málaga: Yunquera; 7.VI.86 (MGC 20681)

Phlomis purpurea L., Málaga: Alhaurín de la Torre; 16.III.86 (MGC 20715)

Platicapnos saxicola Willk., Jaén: Pico de Mágina; 19.VI.83 (MGC 21611)

Platycapnos spicata (L.) Bermh., Málaga: entre Antequera y Lucena; 9.V.88 (MGC 23084)

Platycapnos tenuiloba Pomel. subsp. parallela Lidén, Málaga: Carratraca; 28.IV.88 (MGC 23048)

Platycapnos tenuiloba Pomel. subsp. tenuiloba, Almería: Cabo de Gata; 26.III.84 (MGC 21610)

Ranunculus bullatus L., Málaga: Almogía; 24.X.86 (MGC 21539)

Ranunculus paludosus Poiret, Málaga: Alhaurín el Grande; 16.III.86 (MGC 21626)

Reseda complicata Bory., Granada: Sierra Nevada; 22.VII.87 (MGC 20634)

Reseda phyteuma L., Granada; Motril; 18.IV.86 (MGC 21536)

Rhododendron ponticum subsp. baeticum (Boiss. \& Reuter) Rivas Martínez ex Devesa, Cádiz: Cortes de la Frontera; 8.XI.86 (MGC 18207)

Romulea bulbocodium (L.) Sebastiani \& Mauri, Málaga: Tolox; 5.II.88 (MGC 21548)

Scilla automnalis L. (a), Málaga; Alora; 11.X.86 (MGC 23012)

Scilla autumnalis L. (b), Málaga: Ojén; 8.X.87 (MGC 20651)

Stachys circinata L'Hér, Málaga: Yunquera; 7.VI.86 (MGC 20678)

Teucrium lusitanicum Schreber, Málaga: Alhaurín el Grande; 16.III.86 (MGC 20712)

Teucrium luteum (Miller) Degen, Málaga: Nerja; 17.V.86 (MGC 22990)

Teucrium scordium L. subsp. scordioides (Schreber) Maire \& Petimengin, Huelva: Almonte; 9.IX.87 (MGC 18238)

Theligonum cynocrambe L., Málaga: El Burgo; 27.III.88 (MGC 21559)

Tulipa sylvestris L. subsp. australis (Link) Pamp., Málaga: Alora; 22.III.87 (MGC 23018).

Urginea maritima (L.) Baker, Málaga: Coín; 9.II.86 (MGC 21357)

Valeriana tuberosa L., Málaga: Alora; 22.III.87 (MGC 23014)

Viola crassiuscula Bory., Branada: Sierra Nevada; 22.VII.87 (MGC 20628)

Viscum cruciatum Sieber ex Boiss. (a), Málaga: El Burgo-Ronda; 8.II.86 (MGC 20665)

Viscum cruciatum Sieber ex Boiss. (b), Málaga: Tolox; 24.IV.88 (MGC 23021)

Whitania frutescens (L.) Pauquy., Granada: Calahonda; 28.II.86 (MGC 20703)

Dirección de los autores: R. SUAU, A.I. GARCIA \& R. RICO: Departamento de Bioquímica, Biología Molecular y Química Orgánica, Facultad de Ciencias. Universidad de Málaga. B. CABEZUDO, J.M. NIETO \& E. SALVO: Departamento de Biología Vegetal. Facultad de Ciencias. Universidad de Málaga. 